\title{
Antibiotics Sensitivity Profile Towards Staphylococcus hominis in Assir Region of Saudi Arabia
}

\author{
N. M. Abdalla ${ }^{1 *}$, W. O. Haimour ${ }^{2}$, A. A. Osman ${ }^{3}$, M. A. Sarhan ${ }^{4}$, and H. A. Musaa ${ }^{5}$ \\ ${ }^{1}$ College of Medicine. King Khalid University, Abha, Saudi Arabia \\ ${ }^{2}$ Assir Central Hospital Laboratory Abha, P.O.BOX 1119, Kingdom of Saudi Arabia \\ ${ }^{3}$ Family \& Community Medicine Department, College of Medicine, King Khalid University, 61421 \\ Abha, P.O. 641, Saudi Arabia \\ ${ }^{4}$ Department of Biology, College of Science, King Khalid University, 61413 Abha P.O. Box 9004. \\ Saudi Arabia \\ ${ }^{5}$ Department of Microbiology, The National Ribat University, Khartoum, Sudan \\ Received 13 September 2012, accepted in final revised form 1 November 2012
}

\begin{abstract}
Staphylococcus hominis is a Gram-positive, spherical cells in clusters and coagulasenegative bacterial. It commonly occurs as a harmless commensal on human skin, occasionally causes nosocomial or community acquired infection specially in immunocompromised patients. Nowadays almost all Staphylococcus species have multidrug resistance. In this study, a total of ten out of one hundred fifty nasal swabs at Assir Central Hospital General Lab during the period of April 2011- July 2011 proved to be Staphylococcus spp. and identified as Staphylococcus hominis patients of different sex and age groups with variable systemic infections (e.g. RTI, UTI, CNS). The samples were tested by bactech, culture media, antibiotics sensitivity using diffusion disc test (MIC) and molecular polymerase chain reaction (PCR) for confirmation of Staphylococcal species and detection of the Mec A gene. Clinical, demographic and laboratory data were collected and analyzed by SPSS. Drugs found to be resistant to all patients were penicillin, erythromycin, ampicillin, cifoxine and carbinicillin. Whereas cotrimexazole, amikacine and vancomycine were sensitive to all patients. Only $10 \%$ of patients were sensitive to methotrexate and cefaclor. Drugs that showed variable sensitivity pattern among patients were tetracyclin, fucidin, augmentin, gentamycin and ciprofloxacin.
\end{abstract}

Keywords: Staphylococcus hominis; Coagulase-negative; Multidrug resistance; Grampositive; SHN.

() 2013 JSR Publications. ISSN: 2070-0237 (Print); 2070-0245 (Online). All rights reserved. doi: http://dx.doi.org/10.3329/jsr.v5i1.11704 J. Sci. Res. 5 (1), 171-183 (2013)

\section{Introduction}

Staphylococcus hominis is a coagulase-negative member of the bacterial genus Staphylococcus, consisting of Gram-positive, spherical cells in clusters. Colonies of $S$.

\footnotetext{
*Corresponding author: uofgnazar@gmail.com
} 
hominis are small, usually 1-2 mm in diameter after 24 hours' incubation in blood agar media at $35{ }^{\circ} \mathrm{C}$, white or tan in colour. Occasional strains are resistant to novobiocin and may be confused with other resistant species (e.g. S. saprophyticus.). It is one of only two species of Staphylococcus that display sensitivity to desferrioxamine, the other being $S$. epidermidis. Unlike $S$. epidermidis, S. hominis produces acid from trehalose, so the two tests together serve to identify the species. Numerous coagulase-negative staphylococci appear commonly on human skin, of these species, Staphylococcus epidermidis and $S$. hominis are the most abundant. While S. epidermidis tends to colonize the upper part of the body, $S$. hominis tends to colonize in areas with numerous apocrine glands, such as axillae and the pubic region. S. hominis is able to produce acid aerobically from glucose, fructose, sucrose, trehalose and glycerol. Some strains were also able to produce acid from turanose, lactose, and galactose, melezitose, mannitol, and mannose. The cell wall contains low amounts of teichoic acid and glutamic acid. The cell wall teichoic acid contains glycerol and glucosamine [1].

Based on a total of 240 strains, all were resistant to lysozyme, some were slightly resistant to lysostaphin, $77 \%$ were susceptible to penicillin G, $97 \%$ to streptomycin, $93 \%$ to erythromycin, $64 \%$ to tetracycline, and $99 \%$ to novobiocin [2].

When grown in agar cultures, colonies are usually circular, 4.0 to 4.5 micrometers in diameter. Agar colonies usually have wide edges and an elevated center. They are commonly smooth with dull surfaces, and are yellow-orange pigmented in the center of the opaque colonies. They grow both in aerobic and anaerobic conditions, but tend to grow significantly less in the latter. Optimal $\mathrm{NaCl}$ concentrations of the agar culture for the growth of $S$. hominis seems to be around $7.5 \%$, and a salt concentration of $15 \%$ yielded poor growth to no growth at all. The optimal growth temperature range was around 28 to $40{ }^{\circ} \mathrm{C}$, but good growth is still observed at $45{ }^{\circ} \mathrm{C}$, while no growth is observed at $15{ }^{\circ} \mathrm{C}$. S. hominis can be differentiated from staphylococci by its colony morphology and pigmentation patterns, predominant tetrad cell arrangement, poor growth in thioglycolate, low tolerance of $\mathrm{NaCl}$, and carbohydrate reaction pattern. Each species is also significantly different in cell wall composition, lactic acid configuration, temperature extremes of growth, coagulase activity, hemolysis acetylmethylcarbinol production, nitrate reduction, and phosphatase, DNase, and bacteriolytic activities. Similarities in these properties between $S$. hominis and several other species suggest there is a close relationship between $S$. hominis and S. epidermidis, S. haemolyticus, and S. warneri.[3].

Assir Central Hospital is almost 600 bedded and it is accredited from the Central Board of Arab Health. Moreover it is the only tertiary care facility in the southern part of the Kingdom of Saudi Arabia, with a population of well over a million. The laboratory is a regional referral hub that serves the hospital as well provides consultation for the rest of the region. On the other hand, the hospital is affiliated to the Medical College of King Khalid University. This study is aimed at evaluating the commonly used antibiotics resistant to Staph. non aureus species (hominis, epidermidis) isolates from nasal swabs of patients available at Assir Central Hospital General Lab. 


\section{Material and Methods}

\subsection{Collection of samples}

10 cases of Staphylococcus hominis out of 150 patients with detection of Staphylococcus species directly from nasal swab specimens using bacteriological and molecular methods from clinical isolates presented with variable nosocomial or community acquired infections such as respiratory infection, central nervous system infections, urogenital infection, musculoskeletal (joints) and skin infections were collected from Assir Central Hospital, Saudi Arabia during the period from April 2011- July 2011.

Clinical data including the inpatient and outpatient categories and patient's history of diabetes were used. Then from each patient had a specimen collected from the nares with a dry, unmoistened swab. The tip of the collection swab was inserted approximately 1 in. $(2.56 \mathrm{~cm})$ into the nares and rolled five times in each nostril. Collected specimens were transported and stored at room temperature. Cultures were inoculated and specimens were processed for PCR analysis. Each sample was examined using the procedure described below:

\subsection{Microbiological tests}

The cultures were carried out on blood agar. The plates were incubated for 24 to $48 \mathrm{hrs}$. at $35 \mathrm{C}^{\circ}$ and examined for growth. After incubation each plate was examined to observe the characters of colonial morphology, and the effect of the organism on culture media. The colonies appeared as medium to large, smooth, entire, slightly raised, translucent, most colonies pigmented creamy yellow, most colonies showed beta-hemolysis. Confirmation of Staphylococcus species were conducted using microscopic examination of gram stained film, 3\% catalase testing and coagulase testing according to the working steps of Kayser, Medical Microbiology (C) 2005 Thieme.

\subsection{Kirby-Bauer and Stokes' method (disc diffusion method)}

The Kirby-Bauer and Stokes' methods are usually used for antimicrobial susceptibility testing, with the Kirby-Bauer method being recommended by the NCCLS. The accuracy and reproducibility of this test are dependent on maintaining a standard set of procedures as described here. NCCLS is an international, interdisciplinary, non-profit, non-governmental organization composed of medical professionals, government, industry, healthcare providers, educators etc. It promotes accurate antimicrobial susceptibility testing (AST) and appropriate reporting by developing standard reference methods, interpretative criteria for the results of standard AST methods, establishing quality control parameters for standard test methods, provides testing and reporting strategies that are clinically relevant and cost-effective

Interpretative criteria of NCCLS are developed based on international collaborative studies and well correlated with MIC's and the results have corroborated with clinical 
data. Based on study results NCCLS interpretative criteria are revised frequently. NCCLS is approved by FDA-USA and recommended by WHO (World Health Organization),

\subsection{Preparation of Müeller-Hinton agar culture plates}

An even distribution of the dissolved and mixed Staphyloccus hominis culture colonies in normal saline (1 colony/1 ml of Nacl) was spread on Müeller-Hinton agar plates. The inoculated disks were incubated at 30 to $35^{\circ} \mathrm{C}$ for 24 hours or longer (up to $72 \mathrm{hrs}$ ). The antibiotics disc filter paper was applied over the growing culture colonies again incubated at 30 to $35^{\circ} \mathrm{C}$.

\subsection{Preparation of antibiotics dried filter paper discs}

Whatman filter paper no. 1 is used to prepare discs approximately $6 \mathrm{~mm}$ in diameter, which are placed in a Petri dish and sterilized in a hot air oven. The loop used for delivering the antibiotics is made of 20 gauge wire and has a diameter of $2 \mathrm{~mm}$. This delivers $0.005 \mathrm{ml}$ of antibiotics to each disc.

\subsection{Reading plates and interpreting results}

After 16 to 18 hours of incubation, each plate is examined. If the plate was satisfactorily streaked, and the inoculum was correct, the resulting zones of inhibition will be uniformly circular and there will be a confluent lawn of growth which reflects the minimum inhibitory concentration (MIC). The diameters of the zones of complete inhibition (as judged by the unaided eye) are measured, including the diameter of the disc. Zones are measured to the nearest millimeter, using slide calipers or a ruler. Minimum inhibitory concentration breakpoints of the NCCLS M100-S12: Performance standards for antimicrobial susceptibility testing: Twelfth informational supplement and the organisms are reported as either susceptible, intermediate, or resistant to the agents that have been tested. Some agents may only be reported as susceptible, since only susceptible breakpoints are given.

\subsection{Zone diameter interpretive criteria}

The antimicrobial agents suggested for routine testing of Staph. spp. are indicated below. Each zone size is interpreted by reference to the zone diameter interpretative standards and equivalent minimum inhibitory concentration breakpoints for Staph. spp. The breakpoints for $S$. aureus are different from those for coagulase-negative staphylococci (CoNS). The zone diameter interpretative standards and equivalent minimum inhibitory concentration (MIC) breakpoints for CoNS Staph. spp. for oxacillin sensitivity pattern in CoNS: Susceptible pattern; minimum inhibitory diameter was more than $18 \mathrm{~mm}$ and the minimum antimicrobial concentration was less than $0.25 \mu \mathrm{g} / \mathrm{ml}$ to be considered as sensitive antibiotic while for resistant antibiotic MIC was less than $17 \mathrm{~mm}$ and the minimum antimicrobial concentration was more than $0.5 \mu \mathrm{g} / \mathrm{ml}$. 


\section{Polymerase Chain reaction $(\mathrm{PCR})$}

A nasal specimen is collected and transported to the laboratory using the recommended swab with Liquid Stuart Medium. For testing, the swab was placed in sample buffer. The specimen is concentrated and lysed. An aliquot of the lysate is added to PCR reagents which contain the species-specific primers used to amplify the genetic target, if present. The assay also includes an internal control (IC) to detect PCR inhibitory specimens and to confirm the integrity of assay reagents. The amplification, detection and interpretation of the signals are done automatically by the Cepheid Smart Cycler ${ }^{\circledR}$ software.

\subsection{Molecular analysis}

PCR steps includes: Specimen handling: $24-36$ hours at $15-30{ }^{\circ} \mathrm{C}$ up to 5 days.

Extracting: The specimen swab was broken in buffer tube (blue color, enclosed in extraction kit) and vortex for 60 seconds.

Concentration/Wash: Transfer supernatant to lysis tube (yellow color), centrifuge 5 minutes at $14000 \mathrm{Xg}$ and discard supernatant carefully.

Lysis: Add 50ul of sample buffer (separate tube) to pellet, vortex 5 minutes, quick spin to bring liquid to bottom of tube, heating block at $95{ }^{\circ} \mathrm{C}$ for 2 minutes and put on ice or cooling block or in freezer.

Reagent reconstitution: Add $255 \mu \mathrm{l}$ of diluent to MM tube, vortex 5-10 sec, add $225 \mu \mathrm{l}$ of sample buffer to PC tube (PC: Positive control), vortex 5-10 sec. Aliquot 25ul of MM to SC tubes (SC: Smart cycler) on SC cooling block. Addition of sample (Lysate): Add 2.8ul of lysate to SC sample tubes, addition of Controls, add $2.8 \mu \mathrm{l}$ from kit-PC to new empty PC tube, add $2.8 \mu \mathrm{l}$ of sample buffer to new NC tube (NC: Negative control), centrifuge 5-10 seconds on the SC centrifuge.

Amplification and Detection: Smart Cycler ${ }^{\circledR}$ instrument was used which enclosed the PCR master mix steps: Reconstitute 1 master mix (white label enclosed in the master mix kit) each 1 master mix for up to 6 specimens and 2 controls by adding diluents to lyophilized master mix (A) and transfer master mix to the reservoir of each reaction tube (C); then the lysate solution (B) was transferred to each specimen reaction tube (C). The control DNA was added to positive control tube and sample buffer (blue cap) was added to negative control tube. All tubes were centrifuged briefly. The tubes were placed on specially adapted cooling block till ready to load.

Real-time PCR Analysis: Approximately $5 \mathrm{ng}$ of DNA was added per PCR mixture. The mixture consisted of a buffer system containing $10 \mathrm{mM}$ Tris- $\mathrm{HCl}(\mathrm{pH} \mathrm{9.0),} 50 \mathrm{mM}$ $\mathrm{KCl}, 2.5 \mathrm{mM} \mathrm{MgCl}_{2}, 0.01 \%$ gelatine, and $0.1 \%$ Triton $\mathrm{X}-100$. Deoxyribonucleotide triphosphates (0.2 mM; Pharmacia Biotech, Uppsala, Sweden) as well as $0.2 \mathrm{U}$ of Taq polymerase (SuperTaq; HT Biotechnology, Cambridge, United Kingdom) were present in the reaction mixture. Species specific primers were used in the assays [4]. The codes and species detecting primers (50 pmol of primer per reaction) were as follows: ERIC-1R, 59-ATG TAA GCT CCT GGG GAT TCA C-39; ERIC-2, 59-AAG TAA GTG ACT GGG 
GTG AGC G-39; and the Mec A gene universal primers: P1 5'-GATATGCTTCTCC, P2 5'-AACGTTTAGGCCCATACACCA with amplicon product of $16 \mathrm{bp}$. The PCR mixture was overlaid with $100 \mathrm{ml}$ of mineral oil to prevent evaporation. Amplification of DNA fragments was performed in a Biomed thermocycler (model 60; Biomed, Theres, Germany) with pre-denaturation at $94{ }^{\circ} \mathrm{C}$ for $4 \mathrm{~min}$, followed by 40 cycles of 1 min at 94 ${ }^{\circ} \mathrm{C}, 1 \mathrm{~min}$ at $25{ }^{\circ} \mathrm{C}$, and $2 \mathrm{~min}$ at $74{ }^{\circ} \mathrm{C}$. Amplicons were analyzed by agarose gel electrophoresis containing $1 \%$ agarose (Hispanagar; Sphaero Q, Leiden, The Netherlands) in 0.53 Tris-borate-EDTA (TBE) in the presence of ethidium bromide $(0.0 .3 \mathrm{mg} / \mathrm{ml})$ at a constant current of $100 \mathrm{~mA}$ for around 1 hour. After photography (high-speed sheet film 57; Polaroid), one positive control and one negative control must be included in each assay run on the Smart Cycler®.

\section{Data Analysis}

Clinical and Laboratory data were recorded in special formats and analyzed using statistical computer program (SPSS).

\section{Results}

Out of 150 Staphylococcal samples collected from different departments of Assir Hospital, 10 cases (6.6\%) were found to be Staphylococcus hominess. Staphylococcus hominis laboratory findings include; Gram positive cocci, catalase positive, coagulase negative. The colonies that appeared as medium to large, smooth, entire, slightly raised, translucent, most colonies pigmented white color, most colonies showed beta-hemolysis. Confirmation of Staphylococcus hominis species were performed by PCR. All isolates were Meg A gene negative.

Demographic characteristic of the patients: Around $80 \%$ of the patients were Saudi, $10 \%$ were Arab but not Saudi and $10 \%$ were from other nationalities. $30 \%$ of patients' age range from 0 to more than 50 years, $60 \%$ from 16 to 50 years and finally $10 \%$ of them were more than 50 years old. Two third of the patients were male $(60 \%)$ while $40 \%$ were female. Concerning marital status of patients, $40 \%$ were single and $60 \%$ married. All females were non-pregnant and $20 \%$ of all patients were diabetic and $80 \%$ were nondiabetic (Fig. 1). All samples were nasal swabs. The hospital departments from where samples were collected are shown in (Table 1).

Some antibiotics: Penicillin, erythromycin, ampicillin, cifoxine, carbinicillin, cotrimexazole, amikacine, vancomycine, methotrexate, cefaclor, tetracyclin, fucidin, augmentin, gentamycin and ciprofloxacin were tested to find out their sensitivity and resistance to the Staphylococcus hominis organism. The following drugs were found to be resistant to all patients (all age groups, diabetic and non diabetic) these drugs were penicillin, erythromycin, ampicillin, cifoxine and carbinicillin. Drugs found to be sensitive to all patients (all age groups, diabetic and non diabetic) were: cotrimexazole, amikacine and vancomycine. Only $10 \%$ of the patients were sensitive to methotrexate and cefaclor. 


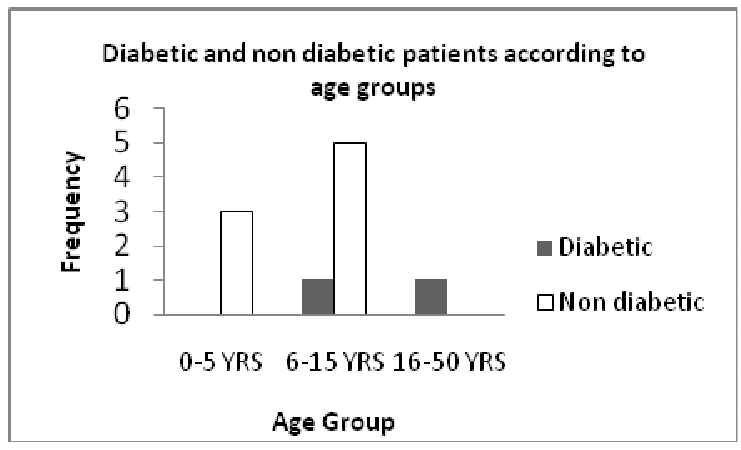

Fig. 1 Diabetic status according to age group.

Table 1. Distribution of samples from different hospital departments.

\begin{tabular}{lll}
\hline Name of department & No. of patients & Percentage \% \\
\hline OPD outpatient department & 2 & 20 \\
ER emergency room & 1 & 10 \\
IMCU intensive medical care unit & 1 & 10 \\
NICU nursery intensive care unit & 1 & 10 \\
MFSW main female surgical ward & 1 & 10 \\
FGS female general surgery & 1 & 10 \\
PICU paediatric intensive care unit & 2 & 20 \\
MOW male orthopedic ward & 1 & 10 \\
\hline
\end{tabular}

Some factors like diabetes and age of the patients were correlated to sensitivity and resistance of the commonly used antibiotics tetracyclin, fucidin, augmentin, gentamycin and ciprofloxacin (Table 2). Augmentin offers resistance to $60 \%$ of non diabetic children as shown in Table 3. Ciprofloxacin sensitivity and resistance were found to be $40 \%, 60 \%$, respectively for different age groups (Table 4). Gentamycin is resistant to all non diabetic children, but it was sensitive in diabetic old patients (Table 5). Tetracyclin is $40 \%$ sensitive and $60 \%$ resistant in different age groups of non diabetic and diabetic patients (Table 6). Fucidin is sensitive in non diabetic adults and diabetic old patients (Table 7). For old diabetic patients the following drugs were sensitive: Augmentin, gentamycin, ciprofloxacin, tetracycline, fucidin and cefaclor. 


\section{Antibiotic Sensitivity}

Table 2. Few commonly used antibiotics resistance and sensitivity pattern.

\begin{tabular}{lllll}
\hline $\begin{array}{l}\text { Name of the } \\
\text { drug }\end{array}$ & $\begin{array}{l}\text { No. of resistant } \\
\text { samples }\end{array}$ & $\begin{array}{l}\text { Percentage } \\
\%\end{array}$ & $\begin{array}{l}\text { No. of sensitive } \\
\text { sample }\end{array}$ & $\begin{array}{l}\text { Percentage } \\
\%\end{array}$ \\
\hline Tetracycline & 5 & 50 & 5 & 50 \\
Fusidin & 6 & 60 & 4 & 40 \\
Augmentin & 7 & 70 & 3 & 30 \\
Ciprofloxacin & 6 & 60 & 4 & 40 \\
Augmentin & 7 & 70 & 3 & 30 \\
\hline
\end{tabular}

Table 3. Augmentin resistance and sensitivity according to age groups and diabetes status of the patients.

\begin{tabular}{llllll}
\hline \multirow{2}{*}{ Age in years } & Diabetes status & & \multicolumn{2}{c}{ Augmentin } & \multirow{2}{*}{ Total } \\
\cline { 3 - 5 } & & sensitive & resistant & \\
\hline \multirow{2}{*}{$0-15$} & Diabetic & 0 & 0 & 0 & 0 \\
\multirow{3}{*}{$16-50$} & Non diabetic & 3 & 1 & 2 & 3 \\
\multirow{5}{*}{50 and more } & Diabetic & 1 & 0 & 1 & 1 \\
& Non diabetic & 5 & 1 & 4 & 5 \\
& Diabetic & 1 & 1 & 0 & 1 \\
\hline
\end{tabular}

Table 4. Ciprofloxacin resistance and sensitivity according to age groups and diabetes status of the patients.

\begin{tabular}{clllll}
\hline Age in years & Diabetes status & \multicolumn{2}{l}{ Ciprofloxacin } & Total \\
& & sensitive & resistant & \\
\hline \multirow{2}{*}{$0-15$} & Diabetic & 0 & 0 & 0 & 0 \\
& Non diabetic & 3 & 1 & 2 & 3 \\
\multirow{3}{*}{$16-50$} & Diabetic & 1 & 1 & 0 & 1 \\
& Non diabetic & 5 & 1 & 4 & 5 \\
50 and more & Diabetic & 1 & 1 & 0 & 1 \\
& Non diabetic & 0 & 0 & 0 & 0 \\
\hline
\end{tabular}


Table 5. Gentamycin, resistance and sensitivity according to age groups and diabetes status of the patients.

\begin{tabular}{llllll}
\hline \multirow{2}{*}{ Age in years } & Diabetes status & & \multicolumn{2}{c}{ Gentamycin } & \multirow{2}{*}{ Total } \\
\cline { 3 - 4 } & & sensitive & resistant & \\
\hline \multirow{2}{*}{$0-15$} & Diabetic & 0 & 0 & 0 & 0 \\
\multirow{2}{*}{$16-50$} & Non diabetic & 3 & 0 & 3 & 3 \\
& Diabetic & 1 & 1 & 0 & 1 \\
50 and more & Non diabetic & 5 & 2 & 3 & 5 \\
& Diabetic & 1 & 1 & 0 & 1 \\
& Non diabetic & 0 & 0 & 0 & 0 \\
\hline
\end{tabular}

Table 6. Tetracycline, resistance and sensitivity according to age groups and diabetes status of the patients.

\begin{tabular}{llllll}
\hline Age in years & Diabetes status & \multicolumn{2}{c}{ Tetracyclin } & \multirow{2}{*}{ Total } \\
\cline { 3 - 4 } & & sensitive & resistant & \\
\hline \multirow{2}{*}{$0-15$} & Diabetic & 0 & 0 & 0 & 0 \\
\multirow{2}{*}{$16-50$} & Non diabetic & 3 & 1 & 2 & 3 \\
& Diabetic & 1 & 1 & 0 & 1 \\
50 and more & Non diabetic & 5 & 2 & 3 & 5 \\
& Diabetic & 1 & 1 & 0 & 1 \\
& Non diabetic & 0 & 0 & 0 & 0 \\
\hline
\end{tabular}

Table 7. Fucidin, resistance and sensitivity according to age groups and diabetes status of the patients.

\begin{tabular}{llllll}
\hline \multirow{2}{*}{ Age in years } & Diabetes status & & \multicolumn{2}{l}{ Fucidin } & \multirow{2}{*}{ Total } \\
\cline { 3 - 4 } & & sensitive & resistant & \\
\hline $0-15$ & Diabetic & 0 & 0 & 0 & 03 \\
& Non diabetic & 3 & 0 & 3 & \\
\multirow{2}{*}{$16-50$} & Diabetic & 1 & 0 & 1 & 1 \\
& Non diabetic & 5 & 3 & 2 & 5 \\
50 and more & Diabetic & 1 & 1 & 0 & 1 \\
& Non diabetic & 0 & 0 & 0 & 0 \\
\hline
\end{tabular}




\section{Discussion}

Few studies on antibiotics sensitivity profile in Saudi Arabia were conducted [5]. In the present study S. hominis clinical isolates count for 6.6\% from 150 Staphylococcal samples collected from Assir region of south-west Saudi Arabia over the of 4 months. In a certain study, S. hominis was calculated to account for $22 \%$ of the total staphylococci species isolated from individuals, second to S. epidermidis at $46 \%$. S. hominis is the predominant species on the head, axillae, arms, and legs. S. hominis is normally found on human skin of usually harmless people, but it can sometimes cause infections in people with abnormally weak immune systems. Most, if not all, strains are susceptible to penicillin, erythromycin, and novobiocin, but a divergent strain, S. hominis subsp. novobiosepticus (SHN) was found recently. The name derives from the combination of novobio, pertaining to the property of novobiocin resistance, and septicus, pertaining to the ability to cause sepsis [6]. This strain failed to produce acid aerobically from trehalose and glucosamine. In addition, the 26 isolated strains of this new subspecies are resistant to nalidixic acid, penicillin G, oxacillin, kanamycin and streptomycin. They were also somewhat resistant to methicillin and gentamicin, and most of them were resistant to erythromycin, clindamycin, chloramphenicol, trimethoprim/sulfamethoxazole and ciprofloxacin, as well. In addition, SHN is not reported to be isolated from the human skin [7]. The SHN is so similar to the original $S$. hominis, now called S. hominis subsp. hominis, that a MicroScan system used by clinical microbiology laboratories can barely differentiate it from $S$. hominis cultures [8].

SHN strains seem to have thickened cell walls, and this tendency may be the result of a genetic background that also allows for vancomycin resistance. The thickened cell walls exist in subspecies with and without vancomycin resistance which suggests this subspecies did not originate from the acquiring of resistance genes. During 2002 and 2003, 32 isolates of SHN were recovered from 21 patients. Eighteen of the 21 patients from which these isolates were recovered were neonates. Among the patients there were one 13-year-old boy, and two were adults. Thirteen of these cases were confirmed as sepsis in neonates resulting from SHN infection. These were the first clinical reports of SHN causing bacterimia in hospitalized patients. Twenty-three isolates were from blood cultures, six were from catheters, one was from cerebrospinal fluid (CSF), one was from a wound, and one was from external ear fluid. All 21 patients yielded an SHN-positive blood culture. All isolates were distinguished from $S$. hominis subsp. hominis on the basis of failure to produce acid aerobically from D-trehalose and resistance to novobiocin. Partial DNA sequencing of the $16 \mathrm{~S}$ rRNA gene from four clinical isolates showed three different PFGE patterns. Out of 21 isolates of SHN from blood, 21 (100\%) were resistant to penicillin, oxacillin, and erythromycin while $20(95.2 \%)$ were resistant to clindamycin and gentamicin, $19(90.5 \%)$ were resistant to tetracycline, $6(28.6 \%)$ were resistant to trimethoprim-sulfamethoxazole, $5(23.8 \%)$ were resistant to chloramphenicol, and 2 (9.5\%) were resistant to ciprofloxacin. All 21 isolates were resistant to nalidixic acid and susceptible to vancomycin. PCR analysis confirmed that all strains possessed a mecA gene 
homologue [9]. These findings are almost similar to the antibiotic sensitivity pattern in our study, as drugs found to be resistant to all patients were: Penicillin, erythromycin, ampicillin. Although all samples showed negative mec A gene by PCR.

The combined resistant to novobiocin and oxacillin is hypothesized to have originated from a simultaneous introduction of genes controlling the resistance of both of them. These genes were believed to have been acquired originally through heterologous DNA from a methicillin-resistant strain of one of the novobiocin-resistant species belonging to the $S$. sciuri or the S. saprophyticus groups. The larger genome size of the SHN compared to that of S. hominis and subsp. hominis may be the result of the acquiring of heterologous DNA. This new, divergent strain was first described in 1998, and this microbe was first implicated in causing bactermia in 2002. Another hypothesis is the insertion of the mec A gene and its flanking sequence into the chromosome of SHN might have affected the expression of a closely linked gene, which converted the host to become novobiocinresistant. The partial 16S rRNA sequence of the clinical isolate of SHN from blood was deposited in GenBank under accession number DQ056840.

SHN infections were high in morbidity, but had a low rate of mortality. A zone of inhibition measuring $\leq 15 \mathrm{~mm}$ in Mueller-Hinton agar or $\leq 11 \mathrm{~mm}$ on Trypticase soya agar plates was considered indicative of novobiocin resistance [10].

All isolates of SHN underwent confirmatory PCR analysis for the mecA gene [11]. To confirm the identification of SHN, broad-spectrum primers were used for sequencing the 5 ' ends of both strands of the 16S rRNA gene [12], using an ABI Prism 3100 genetic analyzer (Applied Biosystems, Foster City, CA). Further molecular characterization of SHN isolates was performed by pulsed-field gel electrophoresis (PFGE) following digestion of DNA extracts with SmaI [13]. More undocumented instances of SHN infections may not have been reported because not all coagulase-negative staphlococcal infections (CONs) are identified to the species level. Molecular epidemiology was successful in identifying emergence of Staphylococcus hominis strains expressing lowlevel resistance to quinupristin/dalfopristin in Greece [14].

Formal investigations regarding the mode of transmission of this microbe were not conducted, but previous study on infants were believed to serve as reservoirs for the microorganism, and transmission takes place with contact between health workers and the infants. In addition, staphylococcal isolates from the nasopharynges and hands of health care workers were shown to be genetically similar to those that colonize or cause disease in neonates [15].

In other studies on neonatal CoNS infections that have demonstrated significant morbidity but a low rate of mortality, there were no deaths associated with SHN sepsis [16]. In one study, 6 out of $13(46 \%)$ neonates with clinically significant bacteremia had proven catheter-related bloodstream infections. CoNS account for a significant proportion of nosocomial bacteremia cases related to the insertion and maintenance of intravascular catheters. Coagulase-negative staphylococci (CoNS) are now recognized as a major cause of nosocomial infective endocarditis in coronary care units (CCU) [17]. 
An important characteristic of SHN is its pattern of multidrug resistance, including resistance to oxacillin. This has important clinical implications because the alternative to oxacillin for treatment of these patients is administration of vancomycin, the overuse of which has implications for the continued emergence of vancomycin resistance in both CoNS and Staphylococcus aureus [18].

\section{Conclusion}

The health workers serve as a form of nosocomical transmission of CONs. If Staphylococcus hominis takes residence on human skin, it probably exists in small numbers and would require enrichment for detection. It has been responsible for nosocomial outbreaks elsewhere. SHN strains have been causing bloodstream infections, but have still been classified as vancomycin-susceptible.

\section{Acknowledgement}

We confer our gratitude to the laboratory of Assir Central Hospital. Our sincere thanks are to the Department of Microbiology, Ribatt National University, Sudan.

\section{Reference}

1. J. Gilad and D. Schwartz, J Clin Microbiol. 45 (2), 685 (2007). http://dx.doi.org/10.1128/JCM.02228-06, PMid:17277175 PMCid:1829067

2. W. E. Kloos, C. G. George, J. S. Olgiate, L. Van Pelt, M. L. McKinnon, B. L. Zimmer, E. Muller, M. P. Weinstein, and S. Mirrett, Int. J. Syst. Bacteriol. 48 Part 3, 799 (1998). http://dx.doi.org/10.1099/00207713-48-3-799 PMid:9734034

3. S. Jiang, B. Z. W. Ding, L. Lv, J. Ji, H. Zhang, Y. Xiao, and L. Li, J. Bacteriol. 2012. 194 (17), 4761 (2012).

4. A. Kilic and A. C. Basustaoglu, Res. Microbiol. 2011. 162 (10), 1060 (2011).

5. N. M, Abdalla, Res. J. Medical Sci. 5 (2.), 94 (2011).

6. A. H. Sohn, D. O. Garrett, R. L. Sinkowitz-Cochran, L. A. Grohskopf, G. L. Levine, B. H. Stover, J. D. Siegel, and W. R. Jarvis, J. Pediatr. 139 (6), 821 (2001). http://dx.doi.org/10.1067/mpd.2001.119442 PMid:11743507

7. I. C. Palazzo, P. A. d'Azevedo, C. Secchi, A. C. Pignatari, and A. L. Darini, J. Antimicrob. Chemother. 62 (6), 1222 (2008). http://dx.doi.org/10.1093/jac/dkn375 PMid: 18775890

8. P. A. d'Azevedo, R.T. T. Sales, J. Monteiro, A. C. Gales, and A. C. Pignatari, J. Med. Microbiol. 57 (Part 2), 256 (2008). http://dx.doi.org/10.1099/jmm.0.47345-0 PMid:18201999

9. F. Chaves, M. G. -A., F. Sanz, C. Alba, and J. R. Otero, J. Clin. Microbiol. 43 (9), 4877 (2005). http://dx.doi.org/10.1128/JCM.43.9.4877-4879.2005

PMid:16145165 PMCid:1234135

10. O. Bouchami, A. B. H, H. de Lencastre, and M. Miragaia, PLoS One 6 (7), e21940 (2011). http://dx.doi.org/10.1371/journal.pone.0021940 PMid:21760926 PMCid:3132775 
11. Y. Katayama, F.T, T. Ito, X. X. Ma, Y. Ui-Mizutani, I. Kobayashi, and K. Hiramatsu, J. Bacteriol. 185 (9), 2711 (2003). http://dx.doi.org/10.1128/JB.185.9.2711-2722.2003 PMid:12700250 PMCid:154413

12. J. E. Fitzgibbon, M. D. N, D. T. Dubin, and J. F. John., Res. Microbiol.152 (9), 805 (2001). http://dx.doi.org/10.1016/S0923-2508(01)01264-5

13. E. Garza-Gonzalez, R.M.-O, M. A. Martinez-Vazquez, E. Gonzalez-Diaz, O. GonzalezSantiago, and E. Rodriguez-Noriega, Scand. J. Infect. Dis. 43 (11-12), 930 (2011).

14. E. Petinaki, I. S, M. Maniati, and A. N. Maniatis, J. Antimicrob. Chemother. 55 (5), 811 (2005). http://dx.doi.org/10.1093/jac/dki072, PMid:15761067

15. A. Sorlozano, J. G, T. Martinez, M. E. Yuste, J. A. Perez-Lopez, A. Vindel, J. Guillen, and T. Boquete, Eur. J. Clin. Microbiol. Infect. Dis. 29 (1), 73 (2011).

http://dx.doi.org/10.1007/s10096-009-0823-4 PMid:19876662

16. W. Le, Curr. Opin. Infect. Dis. 17 (3), 237 (2004). http://dx.doi.org/10.1097/00001432-200406000-00011

17. R. B. Kessler, R. C. Kimbrough, and S. R. Jones, Clin. Infect. Dis. 27 (1), 216 (1998). http://dx.doi.org/10.1086/517680 PMid:9675483

18. D. L. Garza-Gonzaleze, D. L, W. Muruet, V. Bocanegra-Garcia, I. C. Munoz, Ramirez, and L. D. Jm, J. Med. Microbiol. 59, 323 (2010). 